\title{
Implementing and Comparing DSR and DSDV Routing Protocols of Mobile Ad-Hoc Networks using NS2 Simulator
}

\author{
Charu \\ Bhatia \\ Computer \\ Science \\ Department \\ Maharaja \\ Surajmal \\ Institute of \\ Technology \\ Delhi, India
}

\author{
Mona \\ Nandwani \\ Computer \\ Science \\ Department \\ Maharaja \\ Surajmal \\ Institute of \\ Technology \\ Delhi, India
}

\author{
Nidhi \\ Computer \\ Science \\ Department \\ Maharaja \\ Surajmal \\ Institute of \\ Technology \\ Delhi, India
}

\author{
Vandana \\ Negi \\ Computer \\ Science \\ Department \\ Maharaja \\ Surajmal \\ Institute of \\ Technology \\ Delhi, India
}

\author{
Kavita \\ Sheoran \\ Computer \\ Science \\ Department \\ Maharaja \\ Surajmal \\ Institute of \\ Technology \\ Delhi, India
}

\begin{abstract}
MANETs consists of mobile nodes that dynamically form a network temporarily without any support of central administration. MANET is a self organized and self configurable network. Moreover, each node in MANET moves arbitrarily, making routing a crucial issue. This paper presents the performance evaluation of Dynamic Source Routing (DSR) and Destination-Sequenced Distance Vector (DSDV) routing protocols based on metrics such as throughput, packet delivery fraction, dropped packets, packet loss, normalized routing load and average end-to-end delay by using the NS2 simulator.
\end{abstract}

DSDV is a Proactive routing algorithm where the mobile node periodically broadcasts an advertisement message which is transmitted after expiration of the timer.

DSR is a Reactive routing algorithm where a mobile node of MANET connects or advertises the message only when it is needed.

\section{KEYWORDS}

MANET, DSR, DSDV, NS2, Average end-to-end delay

\section{INTRODUCTION}

Ad-hoc is a Latin word, which means "for this or for this only" [1]. An Ad-hoc wireless network which is collection of wireless and mobile nodes that does not rely on a preexisting infrastructure is called Mobile Ad-hoc network. They are selfcreating, self-organizing and self-administrative. Every node becomes a router which must be able to forward the traffic on behalf of other nodes are mobile in nature so their topology is ever-changing and unpredictable that's why "routing" is a major issue periodic or frequent route updates in large MANET may consume significant part of the available bandwidth, increase channel contention and may require each node to frequently recharge their power supply these are the constraints of MANET.

In this paper, DSR and DSDV routing protocols are analyzed on the basis of throughput, packet delivery ratio, average endto-end delay, dropped packets, packet loss and normalized routing overhead and is presented with the simulation results obtained by NS2 simulator.

The organization of rest of the paper is Section II discusses the taxonomy of the routing protocols in MANET. Section III describes DSDV and DSR routing protocols. Section IV describes the performance metrics. Section V describes the simulation of protocols based on the performance metrics. Section VI has Results and Discussion. Finally section VII concludes the paper.

\section{TAXONOMY OF ROUTING PROTOCOLSIN MANETS}

Routing protocols are broadly classified into three types such as Proactive, Reactive and Hybrid protocols [2].

\subsection{Table Driven Protocol}

It is based on the proactive mechanism where each node advertises its routing table to its neighboring nodes periodically or when network topology changes. These protocols maintain the route for all the nodes in their routing tables. In this way each node maintains an up-to-date view of the network. Proactive mechanism works best in the network that have low node mobility or where the nodes transmit data frequently. As the nodes are mobile due to which it will render the routing information in the table invalid quickly. In these protocols packet forwarding is fast on the same side routing overhead is increased as the routing tables have predefined information about all routes in the network. They have lower latency because all the routes are maintained at all the times.

Destination-sequenced distance vector (DSDV) protocol, Optimized Link State Routing (OLSR) come under this class.

\subsection{On-Demand Protocol}

It is based on reactive mechanism where nodes advertise their routing table when it is needed. This will reduce the routing overhead and increase routing latency. In this routing protocol, the route discovery is very important phase where the source node will flood the packets into the network in search of an optimal path to the destination node and also 
updates its routing table. Once a route has been established, some form of route maintenance procedure maintains it until either the destination node becomes inaccessible along every path from the source node or until the route is no longer desired.

Dynamic Source Routing (DSR) protocol, Ad Hoc OnDemand Distance-Vector Routing protocol (AODV) come under this class.

\subsection{Hybrid Protocol}

It is combination of both proactive as well as reactive mechanisms and takes advantages from both of the routing protocols. Due to which it has faster packet forwarding and low routing overhead.

Zone Routing Protocol (ZRP) comes under this class.

\section{OVERVIEW OF DSDV AND DSR ROUTING PROTOCOLS}

This section gives the succinct overview of the routing protocols.

\subsection{Destination - Sequenced Distance Vector (DSDV) Protocol}

DSDV, a table driven protocol, is a modified version of classical Bellman-Ford Algorithm [3]. The tendency of Bellman-Ford algorithm to create routing loops is overcome by adding destination-sequence number field in the routing tables. Each node will transmit the sequence number, which is periodically increased by 2 along with update messages to its neighboring nodes. If the received sequence number is greater than the sequence number stored by the received node, it will update its routing table otherwise it will ignore the update message, thus avoid the formation of loops. Each node maintains its routing table, which consists of list of all available destinations, the hop count and the sequence number. Each node periodically advertises its routing table or if any significant change has occurred in the table to its neighboring nodes, hence updates are both time-driven and event-driven in nature. Two different types of routing updates are there: "full updates" and "incremental updates" [7, 11]. In full update, node advertises the full routing table to all the neighboring nodes whereas in incremental update, only those entries from the routing table are advertised where changes are made, hence congestion is less.

This protocol has four advantages: to avoid loops, avoid count to infinity problem, to reduce high routing overhead and latency for route discovery is low. The disadvantage is huge volume of control messages.

\subsection{Dynamic Source Routing (DSR) Protocol}

DSR, an on-demand routing protocol, utilizes source routing algorithm. This routing protocol uses the cache technology to maintain the routing table. There are two phases in DSR: Route Discovery and Route Maintenance. The node first checks its route cache and then sends the packet. If the route is available in the cache then source node sends the packet through the route to the destination node, else it initiates the Route Discovery process by broadcasting route request (RREQ) packet to the network, to know the route dynamically. Receiving the RREQ packet, node checks its cache. If the received node doesn't have the route to the destination then it appends its own address to the route record field of the RREQ packet, then that packet is forwarded to its neighbors. If the RREQ packet reaches to the destination or to the immediate node that have the route to the destination in its cache then that node generates a route reply (RREP) packet for the source node. When the RREP packet is generated by the destination, it comprises addresses of nodes that have been traversed by the RREQ across the network. Otherwise, the RREP comprises the addresses of nodes the RREQ has traversed concatenated with the route in the intermediate node's route cache. The route carried back by the RREP packet is cached at the source for future use. If any link on the source route is broken in the network then the source node is notified by generating route error (RERR) packet. The source removes any link from its cache by using this packet. DSR makes very aggressive use of source routing and route caching. The advantage of this routing protocol is overhead is low as compared to DSDV by using route cache.

\section{PERFORMANCE METRICS 4.1 Packet Delivery Fraction (PDF)}

PDF is the ratio of data packets delivered to the destination to those generated by the sources [8] and is calculated as follows:

Packet Delivery Fraction=

(Number of Packets Received / Number of Packets Sent) $x$ 100.

\subsection{Average End-to-End Delay (e2e delay)}

It is the average time of the data packet to be successfully transmitted across a MANET from source to destination. The average e2e delay is computed by,

$$
D=\frac{\sum_{i=1}^{n}\left(R_{i}-S_{i}\right)}{n} m \mathrm{sec},
$$

Where $\mathrm{D}$ is the average end-to-end delay, $n$ is the number of data packets successfully transmitted over the MANET, ' $i$ ' is the unique packet identifier, $R_{i}$ is the time at which a packet with unique identifier ' $i$ ' is received and $S_{i}$ is the time at which a packet with unique identifier ' $i$ ' $i$ s sent.

The Average End-to-End Delay should be less for high performance.

\subsection{Packet Loss (PL)}

It is the difference between the number of data packets sent and the number of data packets received. It is calculated as follows:

Packet Loss $=$ Number of data packets sent - Number of data packets received

\subsection{Throughput}

It is the ratio of total amounts of data that reaches the receiver from the source to the time taken by the receiver to receive the last packet. It is represented in packets per second or bits per second [4].

Throughput $($ bits $/ \mathrm{sec})=$

(Number of delivered packets * Packet Size * 8) / Total simulation period

(4) 


\subsection{Normalized Routing Load}

Normalized Routing Overhead is the number of routing packets transmitted per data packet towards destination and calculated as follows [9]:

Normalized Routing Overhead $=$ Number of Routing Packets / Number of Packets Received

\subsection{Dropped Packets}

Dropped data packet also determines network performance. It can be measured as follows [10]:

Dropped Data Packet $(\%)=(($ Number of packets sent number of packets received) / (Total Number of packets sent $) * 100$

\section{SIMULATION}

The simulations were performed using Network Simulator 2 (NS-2.35) [6]. First the tcl file is generated and then using DSDV protocol simulation is done which gives the nam file and trace file. Then another nam and Trace files are created for DSR protocol [5].

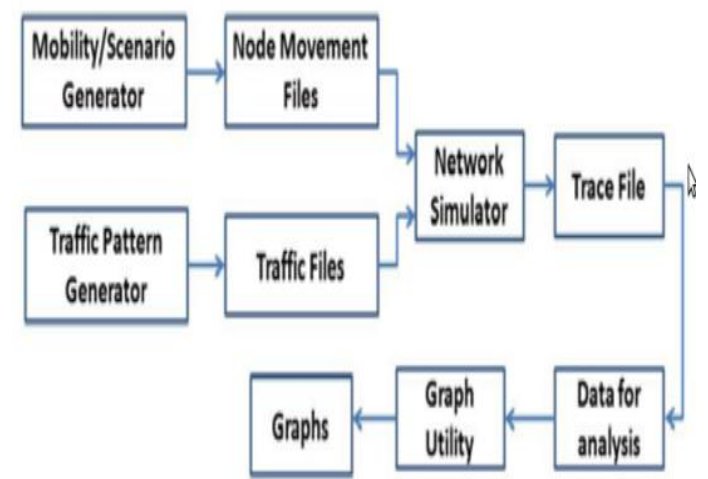

Fig 1: Overview of simulation model

The following table gives the simulation parameters used during the simulation

Table 1: General Simulation Parameters

\begin{tabular}{|l|l|}
\hline Parameter & Value \\
\hline Simulator & NS 2.35 \\
\hline Transmission range & $250 \mathrm{~m}$ \\
\hline Simulation time & $150 \mathrm{~s}$ \\
\hline Routing Protocols & DSDV and DSR \\
\hline Topology Size of mobile & $500 \mathrm{~m}$ X 500 m \\
\hline $\begin{array}{l}\text { Number } \\
\text { nodes }\end{array}$ & $5,10,15,20,25$ \\
\hline Number of sources & $\mathrm{CBR}$ or TCP \\
\hline Traffic Type & 512 bytes \\
\hline Packet Size & $\begin{array}{l}20,40,60,80 \text { and } 100 \\
\text { s }\end{array}$ \\
\hline Pause Time & $\begin{array}{l}2,4,6,8,10 \text { packets } \\
\text { per second }\end{array}$ \\
\hline Network Load
\end{tabular}

\section{RESULTS AND DISCUSSION}

The performance of DSR and DSDV protocol is measured in terms of variation in pause time, number of mobile nodes and network load in CBR traffic.

Table 2(a) shows the observed behavior of DSDV and DSR protocols with respect to varying pause time based on simulation. Table 2(b) shows the observed behavior of DSR and DSDV protocols with respect to varying number of mobile nodes in the simulation process. Table 2(c) shows the observations with respect to changing network load.

Table 2(a): Performance data of DSDV and DSR protocol with respect to pause time

\begin{tabular}{|l|l|}
\hline Performance Parameters & Best protocol \\
\hline Packet delivery fraction & DSR \\
\hline Average end-to-end delay & DSDV \\
\hline Throughput & DSR \\
\hline Dropped packets & DSR \\
\hline Packet loss & DSR \\
\hline Normalized routing load & DSR \\
\hline
\end{tabular}

Table 2(b): Performance data of DSDV and DSR protocol with respect to no. of mobile nodes

\begin{tabular}{|l|l|}
\hline Performance Parameters & Best Protocol \\
\hline Packet delivery fraction & DSR \\
\hline Average end-to-end delay & DSDV \\
\hline Throughput & DSDV \\
\hline Dropped packets & DSR \\
\hline Packet loss & DSDV \\
\hline Normalized Routing Load & DSDV \\
\hline
\end{tabular}

Table 2(c): Performance data of DSDV and DSR protocol with respect to network load

\begin{tabular}{|l|l|}
\hline Performance Parameters & Best Protocol \\
\hline Packet delivery fraction & DSR \\
\hline Average end-to-end delay & DSDV \\
\hline Throughput & DSDV \\
\hline Dropped packets & DSR \\
\hline Packet loss & DSR \\
\hline Normalized routing load & DSR \\
\hline
\end{tabular}

The comparison results of DSR and DSDV protocols are described in terms of six performance metrics by graphs and are discussed below.

\subsection{Packet Delivery Fraction}

Fig 2(a), Fig 2(b) and Fig 2(c) shows the delivery rate of the data packets of DSR and DSDV in terms of variation in pause time, number of mobile nodes and network load. The Packet Delivery rate needs to be high for effective performance of routing. Variation in all the three parameters shows higher values of DSR as compared to DSDV. 


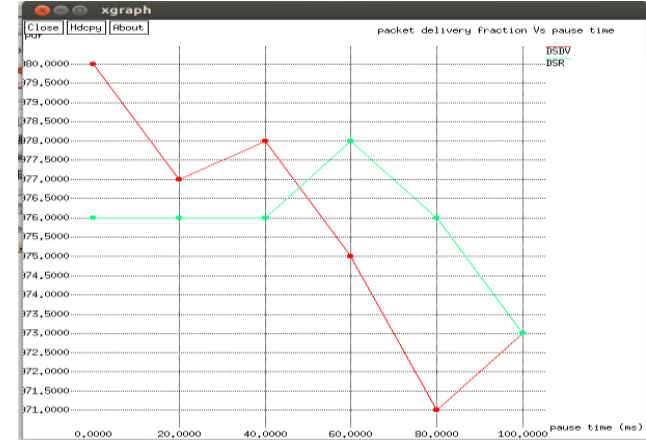

Fig 2(a): PDF of DSR \& DSDV with respect to PT

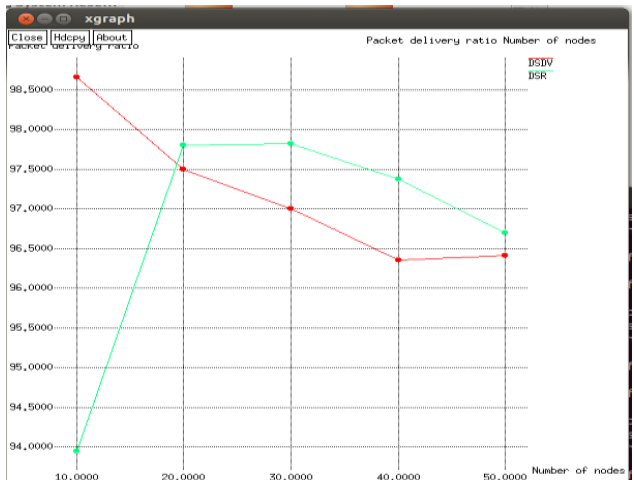

Fig 2(b): PDF of DSR\& DSDV with respect to No. of nodes

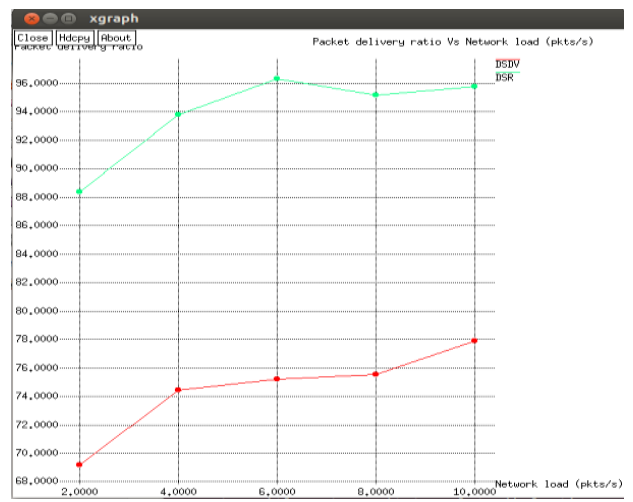

Fig 2(c): PDF of DSR\& DSDV with respect to NL

\subsection{Average End to End Delay}

The End to End Delay is a significant parameter for evaluating a protocol which must be low for good performance. From Fig 3(a), Fig 3(b) and Fig 3(c) the variation in pause time, no. of mobile nodes and network load gives significant impact in DSDV protocol. In other words, the DSDV is performing well than DSR.

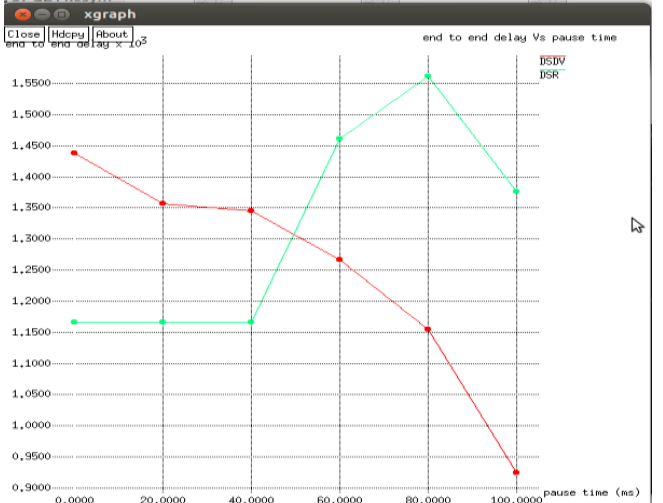

Fig 3(a): e2e delay of DSR \& DSDV with respect to PT

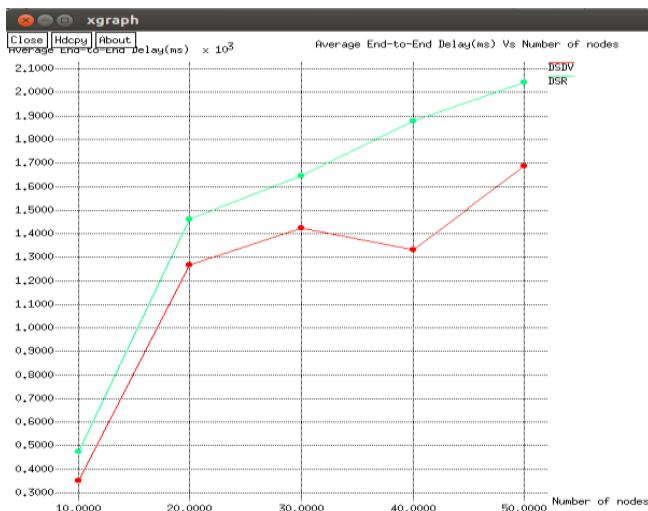

Fig 3(b): e2e delay of DSR \& DSDV with respect to no. of nodes

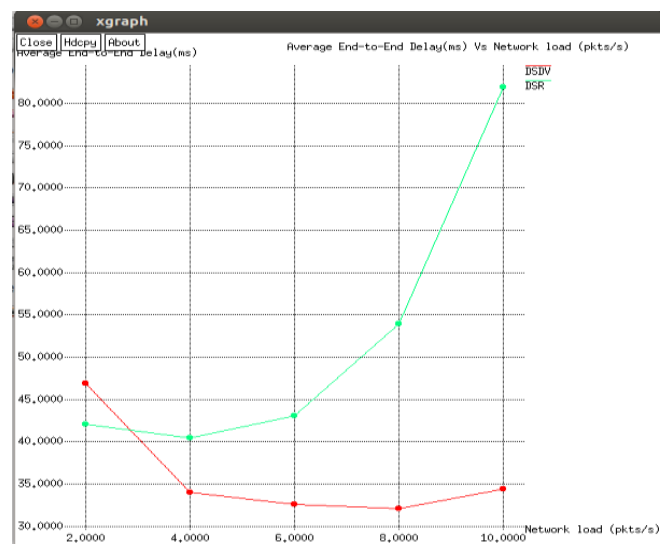

Fig 3(c): e2e delay of DSR \& DSDV with respect to NL

\subsection{Average Throughput}

From Fig 4(a) and Fig 4(b), the variation in pause time and no. of mobile nodes depicts that DSR has better average throughput than DSDV but from Fig 4(c), DSDV shows better result in case of network load. 


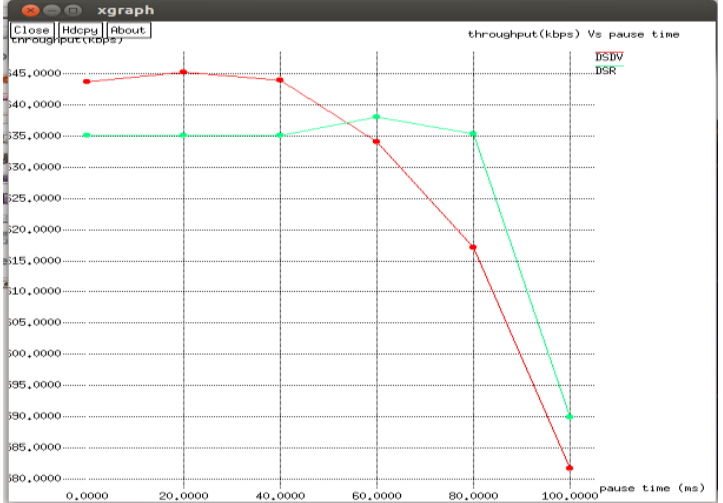

Fig 4(a): Throughput of DSR\& DSDV with respect to PT

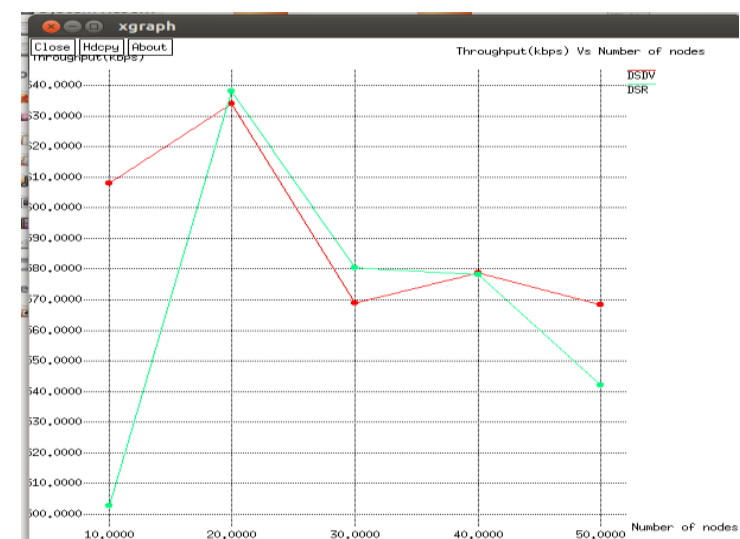

Fig 4(b): Throughput of DSR \& DSDV with respect to no. of nodes

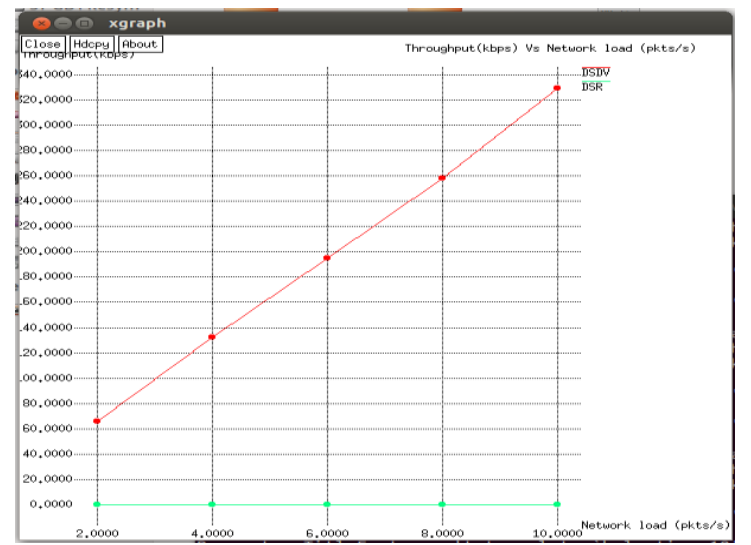

Fig 4(c): Throughput of DSR\& DSDV with respect to NL

\subsection{Packet Loss}

From Fig 5(a), Fig 5(b) and Fig 5(c), the variation in pause time, number of mobile nodes and network load depicts that DSDV has more packet loss than DSR. Packet loss should be less for better performance.

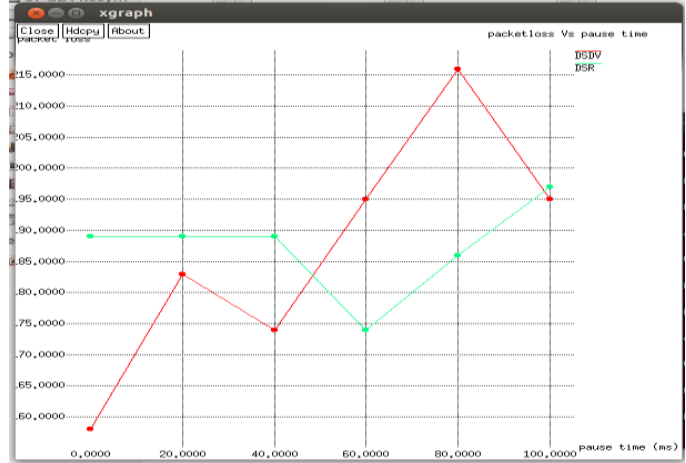

Fig 5(a): Packet Loss of DSR \& DSDV with respect to PT

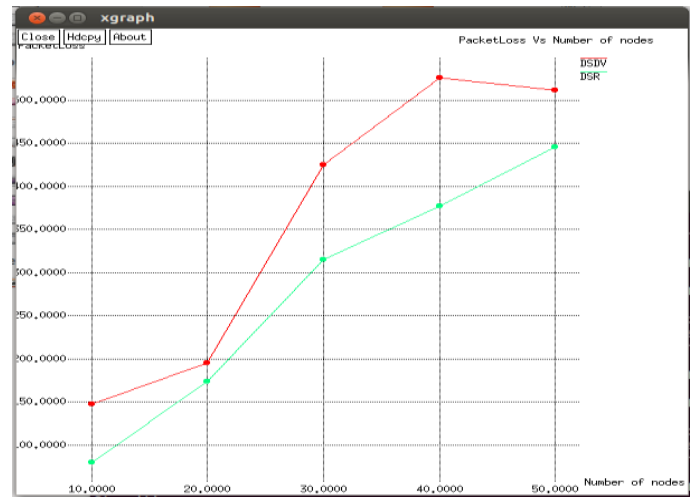

Fig 5(b): Packet Loss of DSR \& DSDV with respect to no. of nodes

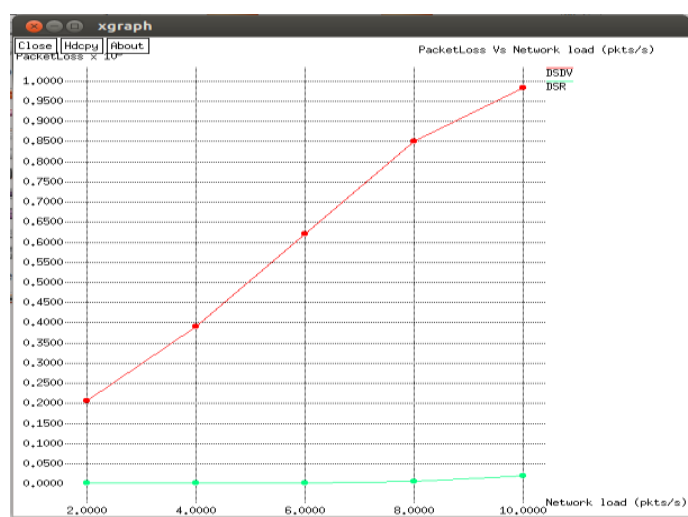

Fig 5(c): Packet Loss of DSR \& DSDV with respect to NL

\subsection{Normalized Routing Overhead}

From Fig 6(a), Fig 6(b) and Fig 6(c), the variation in pause time, number of mobile nodes and network load depicts that DSDV has more normalized overload than DSR. Normalized routing overload should be less for better performance. 


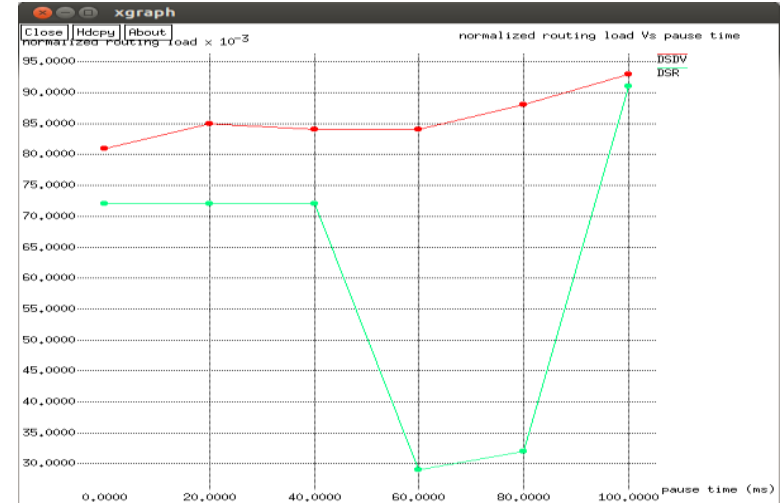

Fig 6(a): Normalized Routing Load of DSR \& DSDV with respect PT

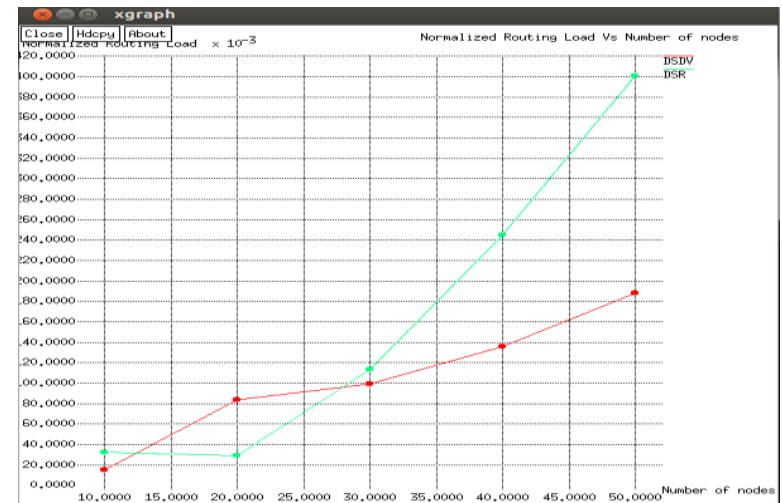

Fig 6(b): Normalized Routing Load of DSR \& DSDV with respect to no. of nodes.

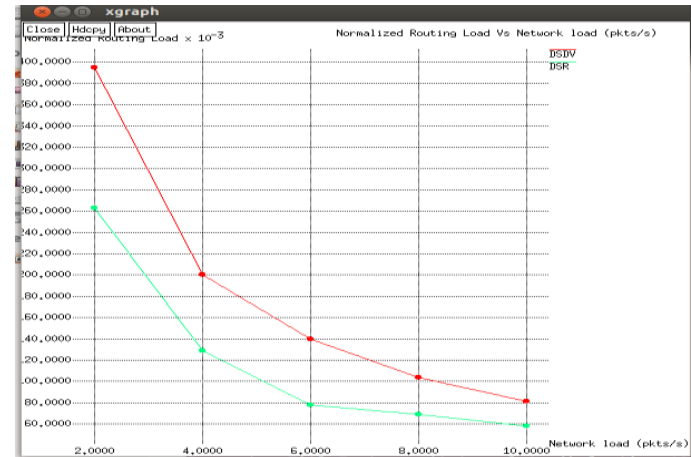

Fig 6(c): Normalized Routing Load of DSR \& DSDV with respect $\mathrm{NL}$

\subsection{Dropped Packet}

From Fig 7(a), Fig 7(b) and Fig 7(c), the variation in pause time, number of mobile nodes and network load depicts that

\section{CONCLUSION}

The DSR and DSDV protocols are compared in terms of the variation in pause time, number of mobile nodes and network load in CBR traffic. Due to randomness in mobility, CBR are selected as scenario parameters. The DSR protocol is giving better performance than the DSDV protocol for most of the performance parametric measures.
DSDV has more dropped packets than DSR. Dropped Packets should be less for better performance.

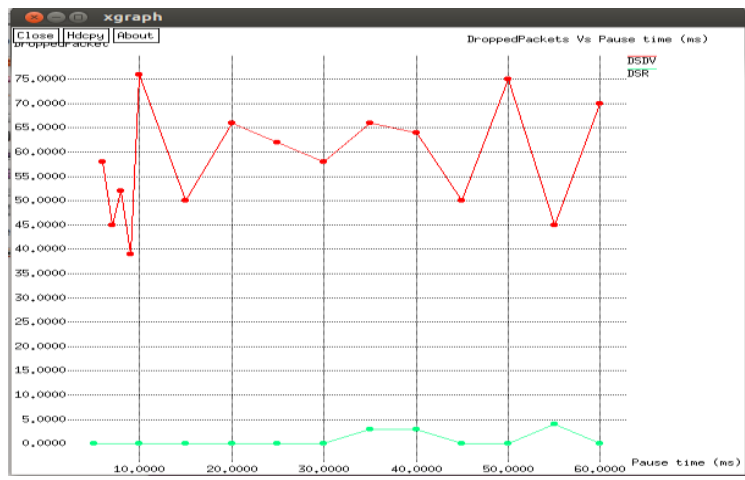

Fig 7(a): Dropped Packets of DSR \& DSDV with respect to PT

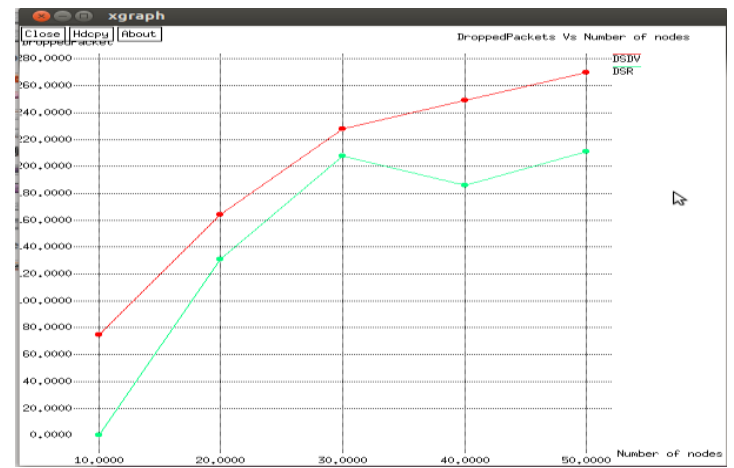

Fig 7(b): Dropped Packets of DSR \& DSDV with respect to no. of nodes

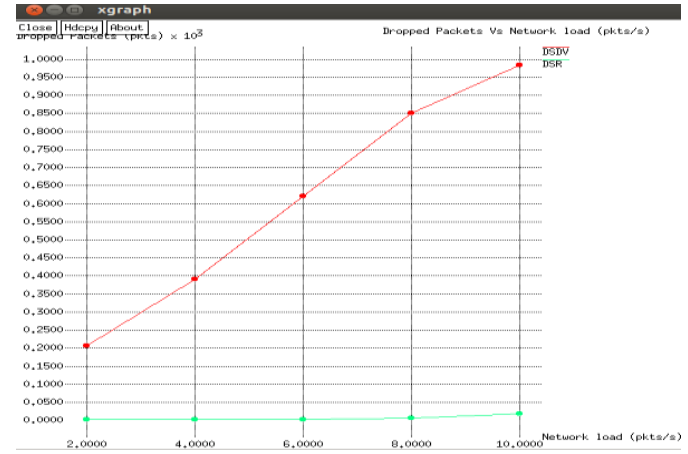

Fig 7(c): Dropped Packets of DSR \& DSDV with respect to NL

\section{REFERENCES}

[1] P. Sharma, Arvind Kalia and Jawahar Thakur "Performance analysis of AODV, DSR and DSDV routing protocols in mobile ad-hoc network (MANET)"Journal of Information Systems and Communication ISSN: 0976-8742 \& E-ISSN: 0976-8750, Volume 3, Issue 1, 2012, pp.-322-326

[2] P. Manickam, T. Guru Baskar, M. Girija, Dr. D. Manimegalai "Performance comparisons of routing protocols in mobile ad hoc networks" - International Journal of Wireless \& Mobile Networks (IJWMN) Vol. 3, No. 1, February 2011 
[3] P. Singh, Dinesh Singh and Vikram Singh "Evaluation of Routing Protocols in MANETs with Varying Network Scope" - 2012 International Conference on Information and Network Technology (ICINT 2012) IPCSIT vol. 37 (2012) (C) (2012) IACSIT Press, Singapore

[4] T. Saleem and Kifayat Ullah "Performance Issues of Routing Protocols in Mobile Ad-hoc Networks".

[5] M.Transier "Ns2 tutorial running simulations"

[6] Network Simulator - ns-2 at http://www.isi.edu/nsnam/ns/

[7] R.P.Yadav, Narendra Singh Yaday, ," Performance Comparison and Analysis of Table-Driven and OnDemand Routing Protocols for Mobile Ad-hoc Networks," Interational Journal of Information and Communication Engineering, 2008

[8] A.Khandakar "Step by Step Procedural Comparison of DSR, AODV and DSDV Routing protocol", Qatar
University 2012 4th International Conference on Computer Engineering and Technology (ICCET 2012) IPCSIT vol.40 (2012) (C) (2012) IACSIT Press, Singapore.

[9] M. tahiliani- Normalized Routing Overhead at http://mohittahiliani.blogspot.in/2011/08/awk-scripts-fornormalized-routing.html

[10] Md. Shohidul Islam, Md. Naim Hider, Md. Touhidul Haque and Letonmiah "An Extensive Comparison among DSDV, DSR and AODV Protocols in MANET", International Journal of Computer Applications (0975 8887)Volume 15- No.2, February 2011

[11] H. Paul and Dr. Prodipto Das "Performance Evaluation of MANET Routing Protocols", IJCSI International Journal of Computer Science Issues, Vol. 9, Issue 4, No 2, July 2012 ISSN. 